\title{
Transseptal Access: A Review of Contemporary Tools
}

Rachel Kaplan ${ }^{1}$, Jeremiah Wasserlauf ${ }^{2}$, and Bradley Knight ${ }^{1}$

${ }^{1}$ Northwestern University Feinberg School of Medicine

${ }^{2}$ Rush University

January 3, 2022

\begin{abstract}
Transseptal left atrial catheterization is routinely used for many common catheter-based interventions. Tools for transseptal catheterization have advanced over the recent years. Such tools include imaging advances with intracardiac echocardiology as well as an array of needles, wires, and dilators to achieve transseptal access with greater ease and safety. This review will discuss the contemporary tools for transseptal catheterization and guidance for difficult cases.
\end{abstract}

\section{Hosted file}

Transseptal review 12.21. docx available at https://authorea.com/users/435918/articles/551376transseptal-access-a-review-of-contemporary-tools

\section{Hosted file}

Video 1.mp4 available at https://authorea.com/users/435918/articles/551376-transseptalaccess-a-review-of-contemporary-tools

\section{Hosted file}

Video 6.mp4 available at https://authorea.com/users/435918/articles/551376-transseptalaccess-a-review-of-contemporary-tools

\section{Hosted file}

Video 2.mp4 available at https://authorea.com/users/435918/articles/551376-transseptalaccess-a-review-of-contemporary-tools

\section{Hosted file}

Video 5.mp4 available at https://authorea.com/users/435918/articles/551376-transseptalaccess-a-review-of-contemporary-tools

\section{Hosted file}

Video 4.mp4 available at https://authorea.com/users/435918/articles/551376-transseptalaccess-a-review-of-contemporary-tools

\section{Hosted file}

Video 3.mp4 available at https://authorea.com/users/435918/articles/551376-transseptalaccess-a-review-of-contemporary-tools

\section{Hosted file}


Video 7.mp4 available at https://authorea.com/users/435918/articles/551376-transseptalaccess-a-review-of-contemporary-tools 